\title{
The aging clock: to 'BMAL'icious toward learning and memory
}

\author{
Jason R. Gerstner \\ Department of Genetics, University of Wisconsin-Madison, Madison, WI 53706, USA
}

\begin{abstract}
Commentary on: Kondratova et al. Circadian clock proteins control adaptation to novel environment and memory formation. Aging. 2010; this issue

Key words: sleep, plasticity, homeostasis, ROS/RNS, chronobiology, consolidation, longevity Correspondence: Jason R. Gerstner, PhD, 3476 Genetics/Biotechnology, 425 Henry Mall, Madison, Wisconsin 53706, USA Received: 05/03/10; accepted: 05/05/10; published on line: 05/07/10

E-mail: jrgerstn@wisc.edu
\end{abstract}

Brain and Muscle Arnt-Like 1 (BMAL1), also known as MOP3 or ARNT3, is a basic helix-loop-helix (bHLH)PAS domain-containing transcription factor that is necessary for the generation of circadian rhythms, and has been implicated in aging. New work shows that BMAL1, or its binding-partner CLOCK, are needed for the generation of new memories. These data suggest a novel molecular link between the processes of circadian rhythms, aging, and memory formation.

It is well known that the process of aging is associated with cognitive decline [1], and disruption in the ability to sleep [2]; recently these features of aging have been shown to be correlated [3]. However, common molecular players that are involved in mediating these processes have not been well characterized. Recent work has begun to establish common molecular mechanisms in the processes of circadian rhythms and memory formation [4], but their relationship to aging so far has not been determined. Now a study by Kondratova et al. [5] in this issue of Aging provides evidence for a core component of the circadian clock, BMAL1, which has previously been shown to significantly influence lifespan [6], in the regulation of learning and memory behavior.

Kondratova et al. [5] examined the role of circadian genes in adaptation, by studying the exploratory behavior and habituation to novelty in various circadian mutant mice, using the open field paradigm. When wild-type (WT) mice are placed in an open field, their novel experience generates an increase in exploratory behavior that begins to decline over time, as the animal remembers the environment (intrasession habituation), and is considered a form of short-term memory (Figure 1A). When WT mice are then placed in this same environment on subsequent days, they exhibit further reductions in exploratory behavior (intersession habituation), which is associated with the formation of long-term memory (Figure 1B). Surprisingly, Bmal1 knock-out (KO) mice display an inability in both intraand intersession habituation (Figure 1C, D), suggesting that Bmal1 gene expression is necessary for both shortand long-term memory.

Next, the authors examined the effects on habituation in mice carrying a mutation for the BMAL1 binding partner CLOCK (Clock/Clock). CLOCK protein forms a heterodimer with BMAL1, and binds to E-box regulatory sequences, in order to drive transcription in the promoter region of downstream target genes, such as Period (Per1,2) and Cryptochrome (Cry1,2), which are also intimately involved in the regulation of circadian rhythms [7-9] (Figure 2). Interestingly, Clock/Clock mice display normal intrasession habituation, but have significantly reduced intersession habituation, suggesting that CLOCK, like BMAL1, is necessary for long-term memory formation. While Clock/Clock mice retain normal short-term memory, the fact that these mice are also deficient in long-term memory formation provides further evidence supporting a role for circadian machinery in the regulation of longterm memory.

Since both Bmal1 KO and Clock/Clock mice display a reduction in intersession habituation, Kondratova et al. 
[5] also examined mice deficient in Cry1,2 (Cry1,2 $\mathrm{KO}$ ) in the open field paradigm. CRY proteins (CRYs) heterodimerize with PER proteins (PERs) to inhibit CLOCK:BMAL1 -mediated transcription, thus generating an autoregulatory negative-feedback loop in the circadian clock (Figure 2). Amazingly, Cry1,2 KO mice not only exhibit both intra- and intersession habituation, but seem to have accelerated habituation; this suggests that Cry1,2 KO mice may have improved short- and/or long-term memory. This interpretation would make sense, given that the Cry1,2 $\mathrm{KO}$ mice likely dis-inhibit CLOCK:BMAL1 transcriptional activity, thereby providing a net increase in downstream target gene expression, such as Per1,2. Overexpression of Per in the fruit fly Drosophila melanogaster has already been shown to enhance long-term memory in courtship conditioning, while Per null flies have significantly impaired memory [10]. Together, these data support a mechanistic relationship for circadian genes in the processes of learning and memory.

Imbalance of reactive oxygen species/nitrogen species (ROS/RNS) homeostasis is associated with aging and cognitive decline [1]. In the current study, Kondratova et al. [5] show that ROS homeostasis is also altered in the brains of Bmal1 KO mice. While levels of ROS do not seem to vary with time-of-day, on average, Bmal1 KO mice have a significant increase in brain ROS as compared to WT, corroborating previous results that have shown increased ROS levels in Bmal1 KO mice with accelerated aging [6]. Interestingly, a recent report found an accumulation of oxidative damage with a significant reduction in lifespan following oxidative stress in Per null flies, as compared to controls [11]. These data support a central role for clock genes in regulating ROS homeostasis, and suggest disruption of circadian pathways results in excessive production of ROS and chronic oxidative stress in the brain.
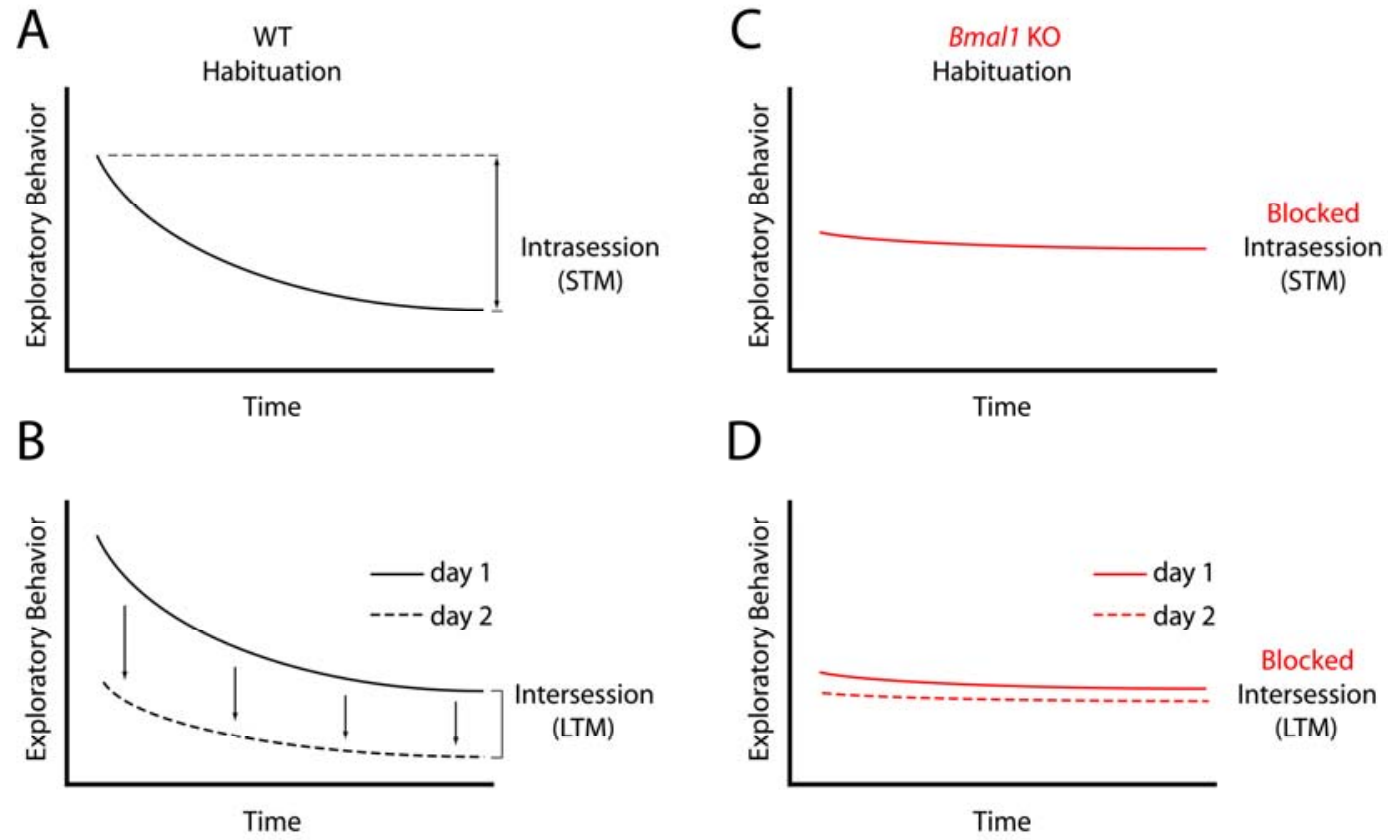

Figure 1. Bmal1 knock-out (KO) mice block habituation memories. (A) Wild-type (WT) mice exhibit a decrease in exploratory behavior in a novel environment over time, called "Intrasession" habituation, a form of short-term memory (STM). (B) Upon reintroduction to the same environment 24 hours later, WT mice have a further decrease in exploratory behavior, attributable to remembering the previous experience. This is called "Intersession" habituation, and is considered a form of long-term memory (LTM). (C) Bmal1 KO mice fail to display normal Intrasession, and (D) Intersession habituation, suggesting deficits in both STM and LTM. 


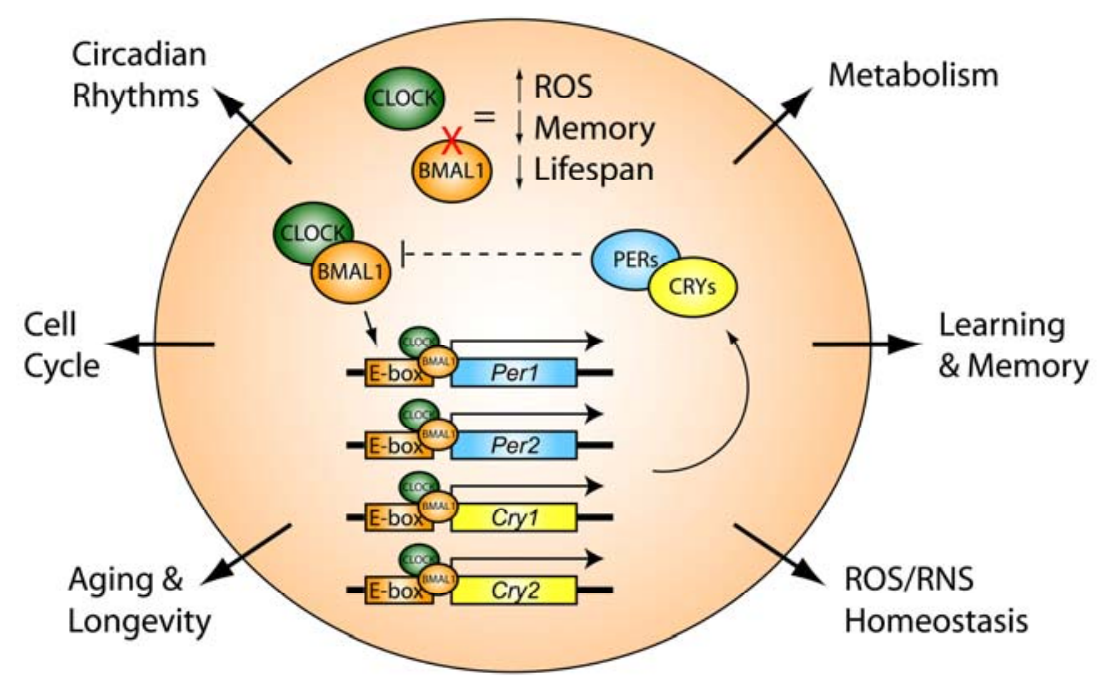

Figure 2. Core components of the circadian transcriptional clock. Brain- and Muscle ARNT-like protein (BMAL1) heterodimerizes with CLOCK protein to bind E-box motifs in the promoter regions of downstream target genes, such as Period (Per 1,2) and Cryptochrome $(C r y 1,2)$ genes. PERIOD proteins (PERs) heterodimerize with CRYPTOCHROME proteins (CRYS) in order to inhibit CLOCK:BMAL1, thus closing an autoregulatory negative-feedback loop. Blocking activity of CLOCK:BMAL1 in Bmal1 knockout mice disrupts normal circadian rhythms, and increases reactive-oxygen species (ROS), while concomitantly decreasing memory and lifespan. Circadian clock output regulates a variety of biological and physiological processes, including circadian rhythms, metabolism, learning and memory, ROS/reactive nitrogen species (RNS) homeostasis, aging and longevity, and the cell cycle.

The circadian clock has been shown to regulate a variety of biological and physiological processes, including sleep/wake rhythms, the cell cycle, metabolism, and aging; and its dysfunction has broad implications in human health $[9,12,13]$. The current study offers further support for the critical involvement of circadian molecules in the regulation of cognitive processing and ROS/RNS homeostasis (Figure 2), providing a molecular link between sleep/wake rhythms, aging, and memory. Loss of the normal sleep/ wake cycle is a primary cause of institutionalization for dementia, and is even thought to be a contributing factor, and/or preclinical sign of neurodegenerative disease, such as Alzheimer's and Huntington's disease [14-16]. For example, in a mouse model of Huntington's disease, which has progressive disruption of sleep/wake rhythms with age [17], reversal of the cognitive decline and survival rate can be achieved by a timed delivery of the benzodiazepine alprazolam, which reinstitutes a daily cycle of sleep $[18,19]$. Observations such as these warrant further study into the functional relationships of other molecular players involved in the circadian clock, especially those that have a role in metabolism, such as PPARs, Rev-erb $\alpha$, and Sirtuins
[13, 20, 21]. Discovering ways that the clock machinery regulates aging, metabolism, the cell cycle, and memory formation (Figure 2) will undoubtedly offer novel therapeutic strategies for the treatment of age-related diseases, such as neurodegeneration, cognitive- and sleep-related disorders, and cancer.

\section{CONFLICT OF INTERESTS STATEMENT}

The author of this manuscript has no conflict of interests to declare.

\section{REFERENCES}

1. Bishop NA, Lu T, Yankner BA. Neural mechanisms of ageing and cognitive decline. Nature. 2010; 464:529-535.

2. Ancoli-Israel S. Sleep and its disorders in aging populations. Sleep Med. 2009; 10 Suppl 1:S7-11.

3. Oosterman JM, van Someren EJ, Vogels RL, Van Harten B, Scherder EJ. Fragmentation of the rest-activity rhythm correlates with age-related cognitive deficits. J Sleep Res. 2009; 18:129135.

4. Gerstner JR, Lyons LC, Wright KP,Jr, Loh DH, Rawashdeh O, Eckel-Mahan KL, Roman GW. Cycling behavior and memory formation. J Neurosci. 2009; 29:12824-12830. 
5. Kondratova AA, Dubrovskaya U, Antoch MP, Kondratov RV. Circadian clock proteins control adaptation to novel environment and memory formation. Aging 2010.

6. Kondratov RV, Kondratova AA, Gorbacheva VY, Vykhovanets $\mathrm{OV}$, Antoch MP. Early aging and age-related pathologies in mice deficient in BMAL1, the core componentof the circadian clock. Genes Dev. 2006; 20:1868-1873.

7. Panda S, Hogenesch JB, Kay SA. Circadian rhythms from flies to human. Nature. 2002; 417:329-335.

8. Gallego $M$, Virshup DM. Post-translational modifications regulate the ticking of the circadian clock. Nat Rev Mol Cell Biol. 2007; 8:139-148.

9. Takahashi JS, Hong HK, Ko CH, McDearmon EL. The genetics of mammalian circadian order and disorder: Implications for physiology and disease. Nat Rev Genet. 2008; 9:764-775.

10. Sakai T, Tamura T, Kitamoto T, Kidokoro Y. A clock gene, period, plays a key role in long-term memory formation in drosophila. Proc Natl Acad Sci U S A. 2004; 101:16058-16063.

11. Krishnan N, Kretzschmar D, Rakshit K, Chow E, Giebultowicz JM. The circadian clock gene period extends healthspan in aging drosophila melanogaster. Aging. 2009; 1:937-948.

12. Reddy $A B, O$ 'Neill JS. Healthy clocks, healthy body, healthy mind. Trends Cell Biol. 2010; 20:36-44.

13. Green CB, Takahashi JS, Bass J. The meter of metabolism. Cell. 2008; 134:728-742.

14. Hatfield CF, Herbert J, van Someren EJ, Hodges JR, Hastings $\mathrm{MH}$. Disrupted daily activity/rest cycles in relation to daily cortisol rhythms of home-dwelling patients with early alzheimer's dementia. Brain. 2004; 127:1061-1074.

15. Hu K, Van Someren EJ, Shea SA, Scheer FA. Reduction of scale invariance of activity fluctuations with aging and alzheimer's disease: Involvement of the circadian pacemaker. Proc Natl Acad Sci U S A. 2009; 106:2490-2494.

16. Gagnon JF, Petit D, Latreille V, Montplaisir J. Neurobiology of sleep disturbances in neurodegenerative disorders. Curr Pharm Des. 2008; 14:3430-3445.

17. Morton AJ, Wood NI, Hastings $\mathrm{MH}$, Hurelbrink C, Barker RA, Maywood ES. Disintegration of the sleep-wake cycle and circadian timing in huntington's disease. J Neurosci. 2005; 25:157-163.

18. Pallier PN, Maywood ES, Zheng Z, Chesham JE, Inyushkin AN, Dyball R, Hastings MH, Morton AJ. Pharmacological imposition of sleep slows cognitive decline and reverses dysregulation of circadian gene expression in a transgenic mouse model of huntington's disease. J Neurosci. 2007; 27:7869-7878.

19. Pallier PN, Morton AJ. Management of sleep/wake cycles improves cognitive function in a transgenic mouse model of huntington's disease. Brain Res. 2009; 1279:90-98.

20. Nakahata Y, Sahar S, Astarita G, Kaluzova M, Sassone-Corsi P. Circadian control of the NAD+ salvage pathway by CLOCK-SIRT1. Science. 2009; 324:654-657.

21. Ramsey KM, Yoshino J, Brace CS, Abrassart D, Kobayashi $Y$, Marcheva B, Hong HK, Chong JL, Buhr ED, Lee C, Takahashi JS, Imai S, Bass J. Circadian clock feedback cycle through NAMPTmediated NAD+ biosynthesis. Science. 2009; 324:651-654. 